\title{
Multitemporal analysis of the flow of sediments using modis MYD09 and MOD09 images
}

\author{
Iván Darío Carrillo Duránª - Rubén Javier Medina Dazab
}

\begin{abstract}
Resumen: en el presente artículo se muestra la elaboración de un modelo empírico aplicado a las imágenes MODIS MYD09 y MOD09 con base en muestras in situ de sólidos suspendidos totales (TSS) que se tomaron en las aguas del golfo de Urabá (Colombia) entre 2011 y 2015. Se analizó la variabilidad espacial y temporal a escala anual y mensual de los sedimentos, lo que mostró una diferencia marcada durante las dos temporadas climáticas (de lluvia y sequia), en la concentración y en la dirección de la pluma de sedimentación. El mejor modelo aplicado sobre las imágenes para la recuperación de los TSS es la forma funcional polinómica de grado 3 que relaciona la sedimentación con la reflectancia de la banda 2, aplicadas en las imágenes MYD09. Dentro de los resultados obtenidos hay valores promedio anuales superiores a los 100mgL-1 en la bahía El Rotico, debido a los aportes dados por el río Atrato en la boca El Roto y de concentración alta de sedimentos en bahía Colombia durante el primer trimestre del año, sobre todo por la dirección de los vientos ( $\approx 150 \mathrm{mgL}-1)$. La investigación permite mostrar las bondades del tratamiento digital de imágenes para recuperar la sedimentación a partir de datos de color del océano, con el propósito de obtener resultados pertinentes que ayuden a realizar un análisis de los flujos de sedimentación en el interior del golfo, sobre todo porque se constituye en un lugar de importancia ecológica por su gran biodiversidad marina y donde el conocimiento de la dinámica y concentración de los sedimentos es escaso.
\end{abstract}

Palabras clave: MODIs; sólidos suspendidos totales; tratamiento digital de imágenes, Golfo de Urabá.

Fecha recepción: 31 de diciembre de 2018 Fecha aprobación: 9 de marzo de 2019

Cómo citar: I.D. Carillo Durán; R.J. Medina Daza. "Multitemporal analysis of the flow of sediments using MODIS MYD09 and MOD09 images", Ciencia e Ingenieria Neogranadina, vol. 29, no. 2, pp. 69-86, 2019. DOI: https://doi.org/10.18359/rcin.3854

a Universidad Distrital Francisco José de Caldas, Colombia.

Correo electrónico: idcarrillod@correo.udistrital.edu.co

b Universidad Distrital Francisco José de Caldas, Colombia.

Correo electrónico: rmedina@correo.udistrital.edu.co 


\begin{abstract}
In this article, we show the development of an empirical model applied to the MODIS MYD09 and MOD09 images based on in-situ samples of total suspended solids (TSS) taken in the waters of the Gulf of Urabá (Colombia) between 2011 and 2015. The spatial and temporal variability at the annual and monthly scale of the sediments was analyzed, showing a marked difference during the two climatic seasons (of rain and drought) both in the concentration and in the direction of the sedimentation plume. The best model applied to the images for the recovery of the TSS was the polynomial functional form of grade 3 that relates the sedimentation with the reflectance of band 2 applied in the MYD09 images. Among the results obtained, it is found that there are annual average values higher than $100 \mathrm{mgL}-1$ in El Rotico Bay due to the contributions given by the Atrato River in the El Roto mouth and high sediment concentration in Bahia Colombia during the first quarter of the year mostly because of the direction of the winds. The research allows us to show the benefits of digital image processing to retrieve ocean color data such as sedimentation, in order to obtain relevant results that allow an analysis of the sedimentation flows within the gulf, especially because it is constituted a place of ecological importance for its great marine biodiversity and where knowledge of the dynamics and concentration of sediments is scarce.
\end{abstract}

Key words: MODIS; Total Suspended Solids; Digital image processing; Gulf of Urabá.

\title{
Introducción
}

Los sólidos suspendidos totales (TSs) son un parámetro clave para evaluar las condiciones de los estuarios y de calidad de las aguas costeras [1], con el fin de realizar el seguimiento del 
transporte y destino de los contaminantes [2]-[4], y determinar el impacto en la disponibilidad de luz dentro de la columna de agua. Esto último afecta directamente la atenuación de la luz y, posteriormente, la producción primaria de plancton, algas bentónicas, pastos marinos y arrecifes de coral [5], [3], [6]-[9]. Los sedimentos en suspensión juegan un papel importante en la gestión de la calidad del agua. La información sobre los Tss a una frecuencia regular es difícil de obtener con datos in situ, ya que los sedimentos no son un parámetro espacialmente homogéneo, lo cual se puede resolver mediante el uso integrado de datos de teledetección, de datos in situ y de modelos de calidad del agua [10].

El aporte de sedimentos por parte del río Atrato es indiscutible, pues su efecto es generalizado y cubre casi la totalidad del golfo de Urabá [11]. Es por ello que la estimación de los tss será posible mediante la elaboración de un modelo que relacione las mediciones in situ tomadas por la Red de Vigilancia para la Conservación y Protección de las Aguas Marinas y Costeras de Colombia (Redcam) con los valores de reflectancia en la imagen Modis, para un periodo de estudio de cinco años comprendido entre 2011 y 2015.

El sensor MODIs reúne unas óptimas características para este objetivo, dada su resolución espacial media, su periodicidad diaria y el libre acceso en línea de los datos [9]. Asimismo, algunos estudios han demostrado que la información de los sensores de satélite del color del océano, como MODIs, representan una alternativa rentable a los métodos tradicionales de muestreo [1], [6].

\section{Teoría del color del océano}

Las propiedades ópticas del mar se dividen en dos grupos: propiedades inherentes y propiedades aparentes [12]. Las primeras son el coeficiente de absorción a, el coeficiente de esparcimiento b y la función de esparcimiento volumétrico $\beta(\theta)$ [12]. Estas magnitudes dependen solo de las sustancias que conforman el medio acuático y no de la estructura geométrica del campo luminoso; las segundas son los coeficientes de atenuación difusa $\mathrm{K}$ y la reflectancia $\mathrm{R}$, entre muchos otros. Estas magnitudes dependen de las propiedades del campo de radiación. La reflectancia $R$ y su variación espectral $R(\lambda)$ pueden vincularse a las propiedades inherentes, siempre que el sol se encuentre lejos del horizonte $\left( \pm 45^{\circ}\right.$ alrededor del cenit), según Jerlov (1976) (citado en [12, p. 32].

Los modelos más aceptados muestran que la reflectancia R se puede expresar de la siguiente forma:

$$
\mathrm{R}=\mathrm{f}\{\mathrm{b} / \mathrm{a}\}
$$

En la ecuación (1), a es el coeficiente de absorción y b es el coeficiente de esparcimiento; por consiguiente, considerando los elementos presentes en el medio, R puede ser tomada como la suma de las propiedades ópticas de la cada uno de estos elementos:

$$
R=f\left\{\frac{\left(b_{w}+b_{p}+b_{s}\right)}{\left(a_{w}+a_{p}+a_{s}+a_{y}\right)}\right\}
$$

En la ecuación (2), $w$ representa el agua de mar, $p$ es el fitoplancton, $s$ son los sólidos suspendidos $\mathrm{y}$, finalmente, $y$ corresponde a la sustancia amarilla.

La teoría del color del mar establece que un sensor remoto óptico localizado a una altura $\mathrm{z} y$ observando a un cierto ángulo recibe una señal $L_{t}$ que puede ser expresada como:

$$
L_{t}=\left(L_{g}+L_{A}+\tau L_{w}\right) * \tau_{g q}
$$

La ecuación (3) posee dos elementos: a. una componente atmosférica constituida por la dispersión de Rayleigh $L_{R}$, la dispersión por aerosoles $L_{A} \mathrm{y}$ la absorción por el ozono $\tau_{o z}$, y b. una componente marina representada por la radiancia emergente del océano $L_{W}$. La diferencia $L_{t}-L_{W}$ se conoce como la componente de la radicación atribuida a la atmosfera y debe ser reducida o eliminada, porque la señal que llega a un sensor remoto está compuesta de una alta contribución (de 85 a $90 \%$ ) y de una pequeña contribución marina de 5 a $10 \%$ (Gordon 1978, citado en [12, p. 33]). Al corregir esta componente se puede obtener la cantidad L_W, que contiene información del cuerpo de agua [12].

\section{Área de estudio}

El área de estudio corresponde al golfo de Urabá (Colombia), el cual comprende desde Cabo 
Tiburón $\left(8^{\circ} 40^{\prime} 34^{\prime \prime} \mathrm{N}, 77^{\circ} 21^{\prime} 32^{\prime \prime} \mathrm{W}\right)$, en la frontera con Panamá, hasta punta Arboletes ( $8^{\circ} 53^{\prime} 18.48^{\prime \prime} \mathrm{N}$ $\left.76^{\circ} 24^{\prime} 58.91^{\prime \prime} \mathrm{W}\right)$, en el límite departamental entre Antioquia y Córdoba (ver Figura 1); limita al norte con el mar Caribe; al sur, con el valle medio del río Atrato; al oriente, con la serranía de Abibe y, al occidente, con la frontera con Panamá, albergando la serranía del Darién [13].

La región del golfo de Urabá presenta un clima húmedo-tropical, y se registra un total anual de precipitación de $2500 \mathrm{~mm}$ aproximadamente. La distribución de la precipitación es monomodal, con un periodo lluvioso que por lo general se establece entre mayo y noviembre $(250 \mathrm{~mm} /$ mes en noviembre) y un tiempo seco desde diciembre hasta mediados de abril (100 mm/mes en febrero). La primera época se caracteriza por alta pluviosidad y la segunda por fuertes vientos alisios de dirección norte-noroeste [13].

\section{Insumos}

\section{Imágenes de satélite}

Se emplearon las imágenes MODIS MYD09GQ Y MOD09GQ para la elaboración y aplicación del modelo diario, las cuales proporcionan una estimación de la reflectancia espectral de la superficie como se mediría al nivel del suelo en ausencia de dispersión o absorción atmosférica, es decir, requiere corrección atmosférica. Tienen una resolución espacial de $250 \mathrm{~m}$, tanto para la banda $1(620-670 \mathrm{~nm})$ como para la banda 2 (841-876 nm), y una resolución temporal de 24 horas. Adicional se utilizó el producto MYD09q1 que cumple las mismas características de las imágenes MYD09GQ y MOD09GQ, pero cada pixel representa el valor de reflectancia en un periodo de ocho días, teniendo en cuenta la cobertura de nueves y el cenit solar para realizar el promedio mensual y anual de Tss. Las imágenes fueron descargadas gratuitamente desde Data Pool (http://e4ftl01. cr.usgs.gov), previo registro en la página de datos de la tierra de la NASA (https://urs.earthdata.nasa.gov/).

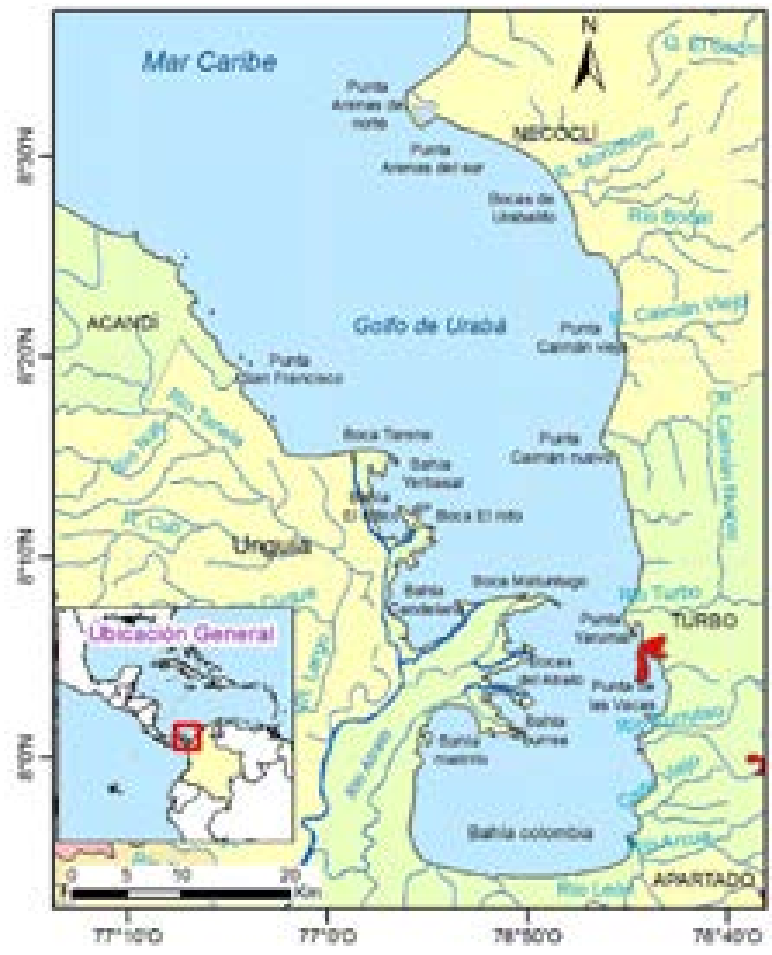

Fig. 1. Mapa de la zona de estudio ubicada en el golfo de Urabá.

Fuente: elaboración propia, a partir de datos del Sistema de Información Geográfica para la Planeación y el Ordenamiento Territorial (SIG-OT). 
Los productos MYD09GQ y MOD09GQ proporcionan una estimación de la superficie de la reflectancia de las bandas 1 y 2 , del sensor Modis Aqua y Terra correspondientemente corregidas por las condiciones atmosféricas, como gases, aerosoles y dispersión de Rayleigh [14] y [15]. Cabe aclarar que las imágenes MODIs con resolución espacial de 250m se seleccionaron en lugar de emplear las imágenes de $500 \mathrm{~m}$ y $1 \mathrm{~km}$, porque, entre otras, las imágenes con menor resolución espacial muestran mejor la variación en una pequeña zona de estudio [16] y también porque muestran una estimación de la reflectancia de la tierra y no es necesario aplicar algoritmos para la corrección atmosférica, ya que es una tarea compleja para llevar a cabo sobre el agua debido a que la señal que sale del agua que alcanza el sensor es muy pequeña en comparación con la señal que llega al sensor de la dispersión atmosférica [17].

$\mathrm{El}$ instrumento MODIs provee una alta sensibilidad radiométrica (12 bit) en 36 bandas espectrales que van desde los $0,4 \mu \mathrm{m}$ hasta los $14,4 \mu \mathrm{m}$; las dos primeras bandas tienen una resolución espacial de $250 \mathrm{~m}$ en el nadir, las siguientes 5 bandas están a $500 \mathrm{~m}$ y las siguientes 29 bandas están a $1 \mathrm{~km}$ [14].

La resolución temporal es diaria, se tienen datos desde julio de 2002 (para Aqua) y desde febrero de 2000 (para Terra); la cobertura de las imágenes es mundial, estas vienen en una proyección sinusoidal y cada imagen cubre una extensión de $1200 \mathrm{~km} \mathrm{x} 1200 \mathrm{~km}$, compuesta de 4800 filas x 4800 columnas, y vienen en el formato HDF [14].

La escena Modis de la zona de estudio está identificada por ser la h10 v08 que tiene longitud mínima de -81,2341, longitud máxima de -69,9917, latitud mínima de 0 y latitud máxima de 10 grados.

El total de las imágenes MYD09Q1 descargadas fue de 231, mientras que se descargaron 1826 imágenes MYD09GQ y 68 imágenes MOD09GQ para los días cuando se habían realizado mediciones de Tss. Todas las imágenes descargadas comprenden el periodo del 1 enero de 2011 a 31 de diciembre de 2015.

\section{Datos in situ de sólidos suspendidos totales}

El monitoreo de calidad de aguas marino-costeras se hace en regiones de importancia ecológica, turística o social, o que presentan tensores como vertimientos directos de aguas residuales domésticas e industriales, inadecuado manejo de basuras, escorrentías agropecuarias, asentamientos humanos, entre otros que pueden afectar la calidad del agua y causar el deterioro de sus ecosistemas [18].

En el Urabá antioqueño la Redcam cuenta con 26 estaciones de muestreo; en todas las estaciones se midieron datos de campo in situ usando equipos portátiles previamente calibrados y se recolectaron muestras de agua para analizar en el laboratorio [18].

Para la determinación de la variable de Tss dada por la Redcam se empleó el método gravimétrico, la unidad de medida empleada es la de mg L-1. Los datos de Tss fueron aportados por la Redcam y en estos se discriminan el nombre de la estación, la fecha y la hora de adquisición de los datos, la localización geográfica de las estaciones y el valor de Tss in situ en mg L-1; además, se cuenta con 236 datos comprendidos entre 2011 y 2015 de 26 estaciones ubicadas a lo largo del golfo de Urabá y boyas cercanas a la desembocadura del río Atrato (ver Figura 2) [19].

De las 26 estaciones, solo 15 se encuentran entre Necoclí y Acandí, las otras pertenecen a la región del Urabá entre Sucre y Punta Caribana, y no se usaron dado que la probabilidad de medición de tss que proviene directamente del río Atrato es muy baja.

\section{Métodos}

Dentro del procesamiento de datos, lo primero que se realizó fue la conversión de las imágenes MYD09GQ con formato HDF de MODIS a TIF. Luego, se reproyectaron las imágenes que provienen de una proyección sinusoidal a una proyección UTM zona $18 \mathrm{~N}$ que corresponde a la zona de estudio. Se mantuvo la resolución radiométrica de 16bits y la misma resolución espacial por píxel se tomó en 250 metros. Se empleó el método de vecino más cercano para el remuestreo, dado que preserva el valor de reflectancia de la imagen original [20] y también para evitar el efecto de adyacencia tierra que induciría valores de reflectancia más altos [21].

Los valores de reflectancia de las imágenes se encuentran en un rango válido de -100 a 16000 [14], y se deben multiplicar por el factor de escala de 0,0001 
[14], lo cual arroja la reflectancia normalizada en un rango válido de -0,01 a 1,6. Se realizó el recorte de la imagen con las siguientes coordenadas UTM ( $\min \mathrm{x}$ $=230802,71, \max x=334417,07, \min \mathrm{y}=869707,74$, max y $=967332,40$ ); este procedimiento se hizo para las 1826 imágenes MYD09GQ, que corresponden al periodo entre el 1 de enero de 2012 y el 31 de diciembre de 2015. Una vez hecho el recorte se les aplicó a las imágenes una máscara de superficie terrestre con el Shapefile de los departamentos del Choco y Antioquia obtenido del SIG-OT, para delimitar el borde costero.

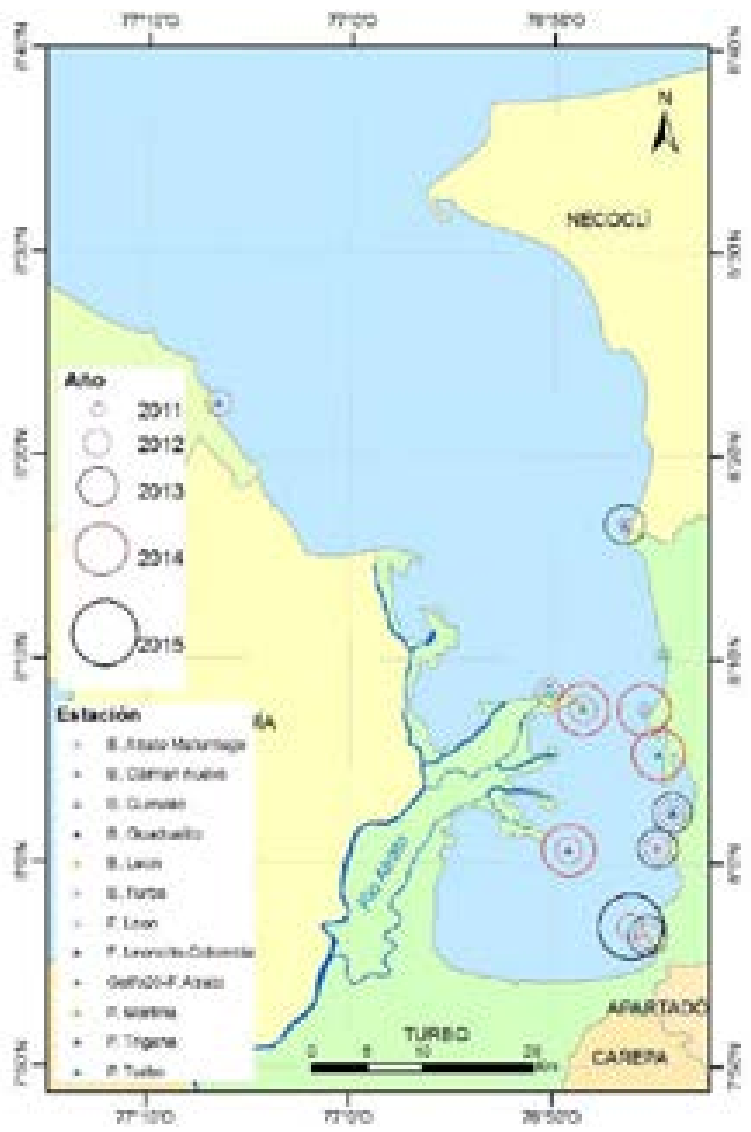

Fig. 2. Ubicación de las estaciones de la Redcam

Fuente: elaboración propia con datos del SIG-OT.

Nota: en esta ubicación se dispone de mediciones in situ de tss para la elaboración del modelo de regresión. En círculos de color negro y rojo se discrimina la disponibilidad temporal de las mediciones.

Luego se escogieron las imágenes con menos del $15 \%$ de nubes, las cuales se identificaron según el porcentaje de píxeles con un valor de reflectancia mayor a 0,3 en la banda 2 (NIR), como realizaron [1], [3], [22] y [23]. El límite de 0,3 se escoge porque la banda 2 detecta muy bien las nubes y porque con altos valores de sedimentación la reflectancia no llegaría a este valor.

La forma de obtener los valores de reflectancia en cada estación fue con la metodología empleada por
[21] y [24], en la que se minimiza el ruido del sensor empleando una máscara de $3 \times 3$ píxeles centrada en el píxel donde se ubica la estación (ver Figura 3). Al menos deben existir 4 píxeles válidos, "el coeficiente de variación debe ser menor a 0.4 " [24, p. 6]; el valor de la mediana de los píxeles válidos se elige como el valor de reflectancia asociado al valor de tss en cada estación [24] y [25]. Otros autores como [25] emplearon máscaras de $5 \times 5$ píxeles, pero este tamaño de máscara no se usó porque varias estaciones 
tomarían valores de reflectancia de tierra dada la proximidad de las estaciones a la línea de costa.

Sin embargo, para mejorar la precisión del modelo se seleccionaron las mediciones in situ en un intervalo de -3,3 horas del paso del satélite [26], para minimizar los efectos de la diferencia temporal entre la recolección de los datos in situ y la captura de las imágenes MODIs [27].

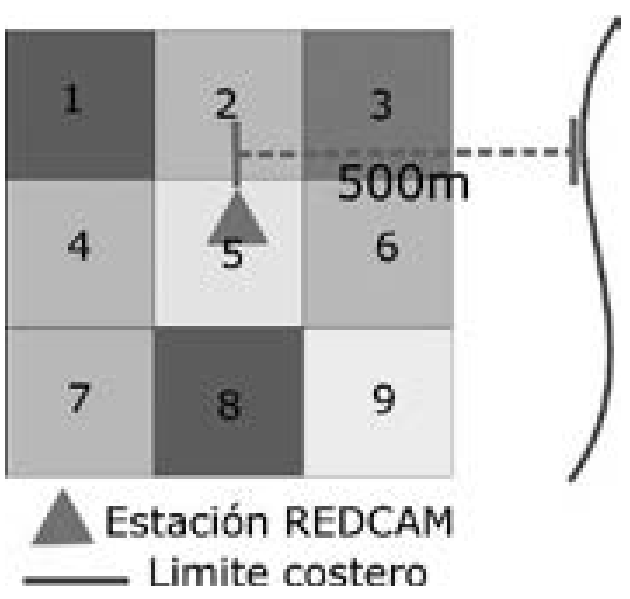

Fig. 3. Esquema de la ubicación de la estación de la Redcam

Fuente: elaboración propia.

Nota: en la figura se muestran los 8 píxeles contiguos, así como de la distancia a la costa para evitar el efecto de adyacencia.

Además, con los datos in situ proporcionados por la Redcam se procedió a verificar los días en los cuales se realizaron más de 5 mediciones, y se verificó que la información recolectada coincidiera con el día que se obtuvieron las imágenes del sensor Modis con un porcentaje de nubosidad menor al $10 \%$. El propósito de esto fue comparar por medio de perfiles de reflectancia los sensores Aqua y Terra para decidir si se pueden utilizar datos de ambos sensores en la modelación de Tss.

Se decide probar varias formas funcionales de modelos de regresión para la elaboración del modelo, como se puede observar en la Tabla 1. Para la evaluación de la calidad se aplicarán los siguientes índices de calidad que han sido empleados por la mayoría de los autores citados en esta investigación.

Raíz del error cuadrático medio RMSE (del inglés root-mean-square error):

$$
\text { RMSE }=\sqrt{\frac{\sum_{i=1}^{i}\left(x_{i}-x_{v}\right)^{2}}{n}}
$$

Error porcentual absoluto medio (MAPE):

$$
\operatorname{MAPE}(\%)=\frac{\sum_{s}\left|x_{1}-x_{4}\right|}{\sum_{i=1} x_{4}} \cdot 100
$$

Índice Bias:

$$
\text { Bias }=\frac{1}{n} \sum_{i=1}^{n}\left(x_{i}-x_{v}\right)
$$

Desviación absoluta media (MAD):

$$
\operatorname{MAD}=\frac{1}{n} \sum_{i=1}^{n}\left|x_{j}-x_{p}\right|
$$

En las ecuaciones $(4,5,6$ y 7$) x_{i}$ es el valor de Tss predicho por el modelo y $x_{v}$ es el valor de Tss in situ. El RMSE puede incrementar si un residual es más alto que otros residuales por el cuadrado del residual, mientras que el MAPE no sufre del problema del cuadrado [28]. Así, teniendo en cuenta la descripción metodológica se ha elaborado el diagrama de flujo de actividades en la Figura 4. 


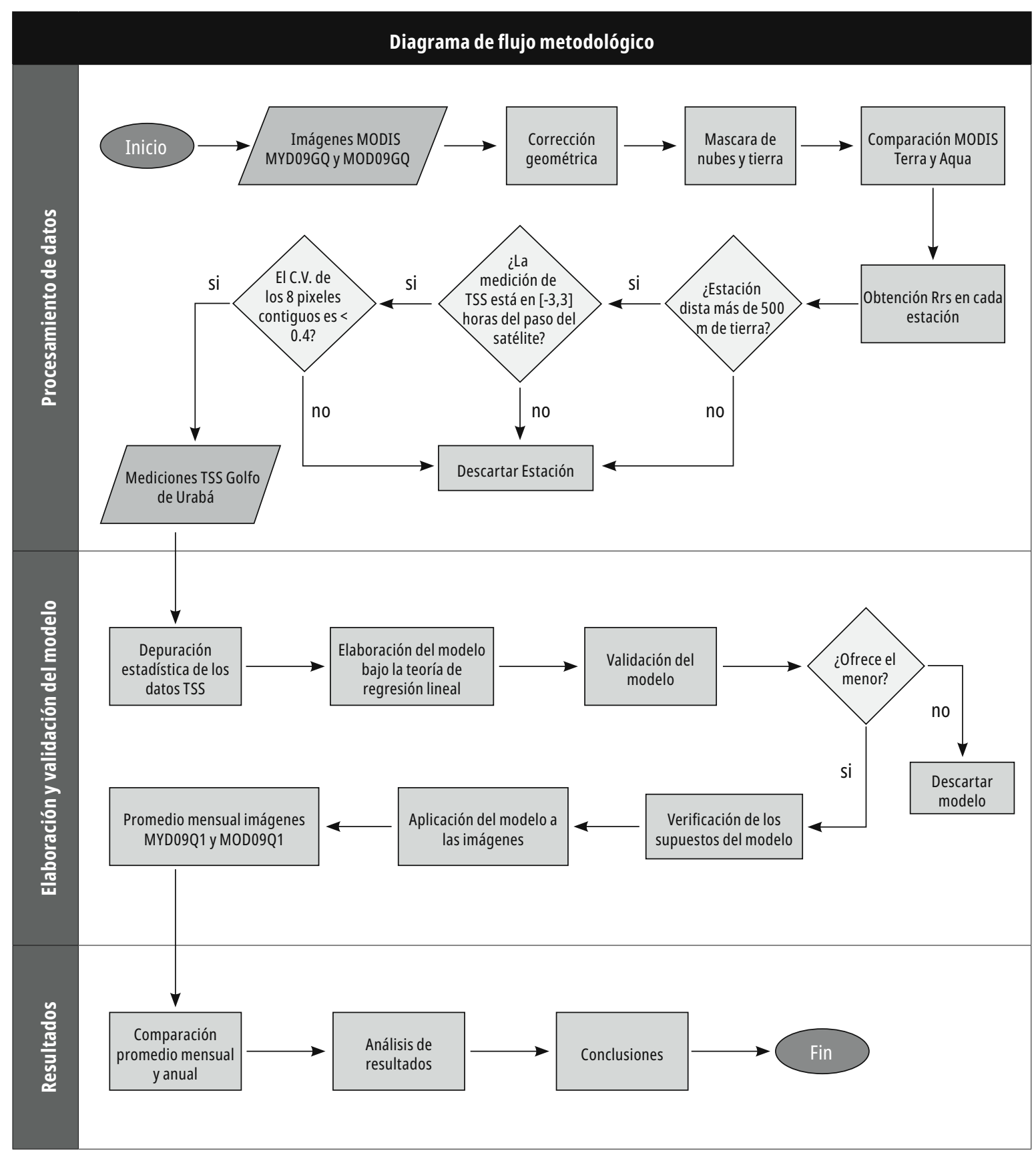

Fig. 4. Diagrama de flujo de las actividades más importantes por realizar en la modelación de sólidos suspendidos totales

Fuente: elaboración propia.

\section{Resultados}

En la Figura 5 se observa la evolución de las mediciones de tss en las 3 estaciones ubicadas en la desembocadura del rio Atrato, debido a que están en un sitio estratégico para medir la sedimentación que llega directamente de este rio.

Además, se procedió a analizar el comportamiento de los valores de reflectancia a lo largo de 3 perfiles espectrales en la desembocadura del río 
Atrato en tres de sus principales bocas, tomando el promedio mensual de noviembre de 2012; esto con el propósito de verificar la concentración de sedimentación a medida que se aleja de la desembocadura del río Atrato, como se observa en la Figura 6.

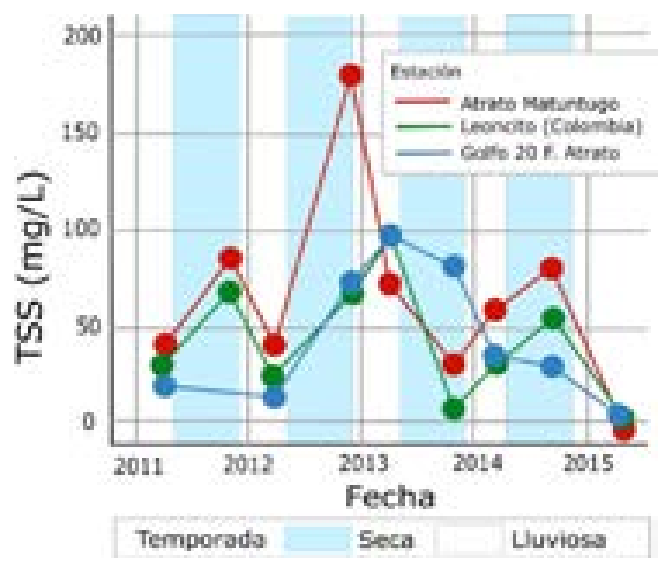

Fig. 5. Mediciones de TSs realizadas en las estaciones ubicadas en la desembocadura del río Atrato Fuente: elaboración propia. Nota: se identificaron tres estaciones en las 3 bocas más importantes del Atrato.

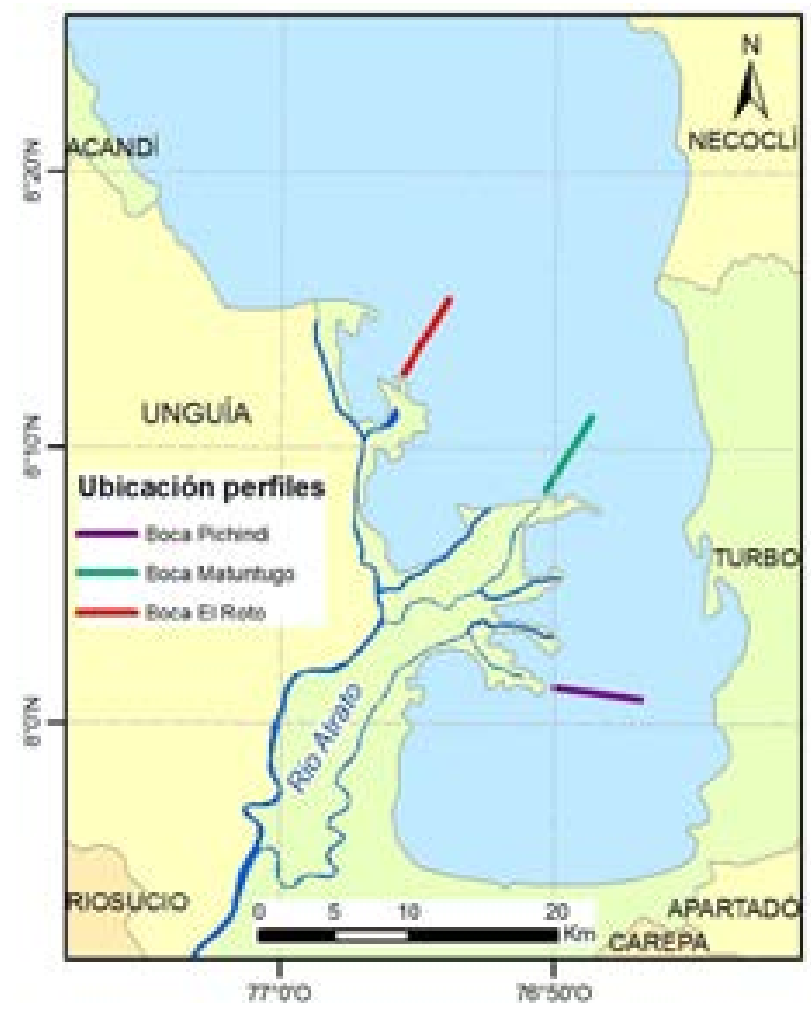

Fig. 6. Ubicación espacial de 3 perfiles espectrales en 3 bocas de la desembocadura del río Atrato Fuente: elaboración propia. Nota: las bocas utilizadas para los perfiles fueron Matuntugo, Pichindi y El Roto. 
En la Figura 7 se ven los perfiles de reflectancia en cada una de las tres bocas analizadas anteriormente, y se observa en la boca Matuntugo un comportamiento similar entre las bandas 1 y 2 de Aqua, y entre las bandas 1 y 2 de Terra; sin embargo, la separación de las curvas es mayor en las bandas del sensor Terra, que llega a ser del $62 \%$ en el primer píxel a diferencia del sensor Aqua, que en el primer píxel tiene una diferencia entre las curvas del $46 \%$. Para los demás píxeles esta separación tiende a disminuir. Para la boca Pichindi se observa un fuerte contraste en las bandas del sensor Terra, debido a los cambios bruscos en los valores de reflectancia (hasta del $90 \%$ ), que no deberían esperarse de un píxel a otro porque la reflectancia en el agua debe cambiar lentamente de un píxel a otro. Una gran diferencia indica un posible efecto de adyacencia tierra [21], mientras que en la boca El Roto se da de nuevo un valor alto de reflectancia en el último píxel de la banda 2 de Terra, que es de 0,16 y la curva de reflectancia muestra desde el píxel 1 hasta 25 una tendencia a aumentar. Este comportamiento es contrario al que se presenta en las otras 3 curvas de reflectancia.
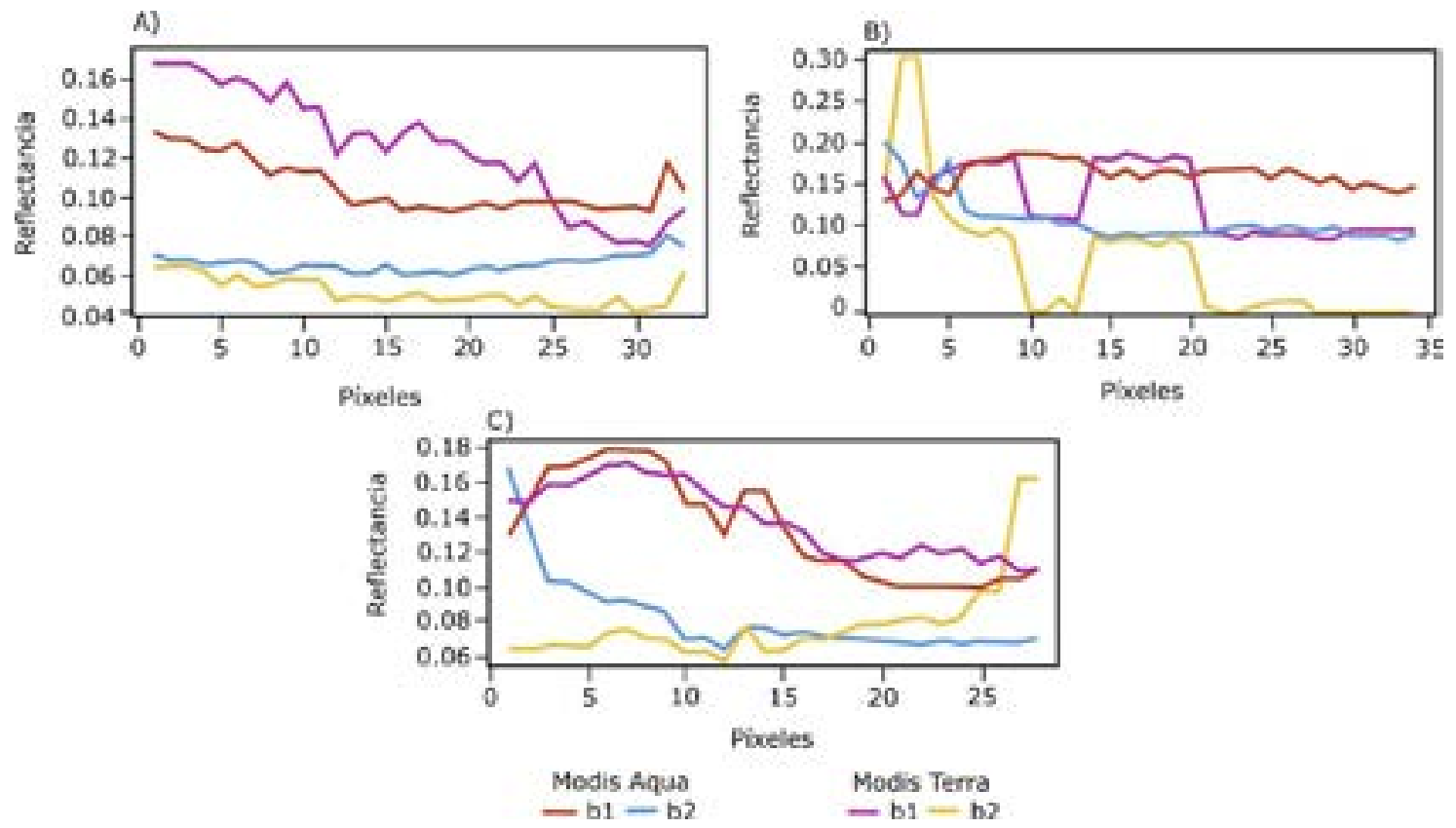

Figura 7. Perfiles de reflectancia para cada una de las bocas en la desembocadura del río Atrato. a. boca Matuntugo; b. boca Pichindi, y c. boca El Roto

Fuente: elaboración propia. 


\section{Elección del modelo}

En la Tabla 1 se muestran, además de los índices de calidad que se presentaron en las ecuaciones $(4,5$, 6 y 7), el coeficiente de correlación de la regresión del modelo ajustado $r^{2}$ y la significancia global del modelo f.

En la Tabla 1 se muestran modelos empleando solo la banda 1 (modelo nro. 1) o solo la banda 2 (modelo nro. 2), combinación de los valores de reflectancia de las bandas 1 y 2 (modelos nros. 4, $5,6,7$ y 9), teniendo en cuenta la suma de bandas (modelo nro. 3), usando la relación exponencial (modelos nros. 8 y 9). Además, varios autores han realizado regresiones polinomiales hasta de orden 3 con la banda 1 y 2 , respectivamente, que toman la siguiente forma:

$$
\mathrm{TSS}=\beta_{0}+\beta_{1}(\mathrm{bl})+\beta_{2}\left(\mathrm{~b} 1^{\wedge} 2\right)+\beta_{3}\left(\mathrm{~b} 1^{\wedge} 3\right)
$$

Por esto se aplica la regresión por pasos "Stepwise" a la ecuación (8), la cual permitirá tener los mejores parámetros para el modelo polinomial. Se aplicó Stepwise a los modelos nros. 10, 11 y 12 mostrados en la Tabla 1.

Tabla 1. Comparación entre los modelos implementados

\begin{tabular}{c|c|c|c|c|c|c|c}
$\mathbf{N}^{\circ}$ & Modelo & $\boldsymbol{r}^{2}$ & $\mathbf{f}$ & $\mathbf{S E}$ & RMSE & MAD & CC \\
\hline 1 & $\mathrm{TSS}=-23.59+767.27(\mathrm{~B} 1)$ & 0,46 & $22.56^{* * *}$ & 27,85 & 26,26 & 19,66 & .69 \\
\hline 2 & $\mathrm{TSS}=-5.34+632.31(\mathrm{~B} 2)$ & 0,51 & $28.03^{* * *}$ & 26,35 & 24,84 & 19,89 & .73 \\
\hline 3 & $\mathrm{TSS}=-39.61+492.84(\mathrm{~B} 1)+447.83(\mathrm{~B} 2)$ & 0,66 & $25.93^{* * *}$ & 21,97 & 20,27 & 17,12 & .83 \\
\hline 4 & $\mathrm{TSS}=60.07+189.77(\mathrm{~B} 2-\mathrm{B} 1)$ & 0 & 1.04 & 37,97 & 35,80 & 26,81 & .20 \\
\hline 5 & $\mathrm{TSS}=-38.70+466.62(\mathrm{~B} 1+\mathrm{B} 2)$ & 0,67 & $53.97^{* * *}$ & 21,52 & 20,29 & 17,12 & .83 \\
\hline 6 & $\mathrm{TSS}=38.38+20.95(\mathrm{~B} 2 / \mathrm{B} 1)$ & 0,01 & 1.49 & 0,23 & 35,49 & 26,38 & .24 \\
\hline 7 & $\mathrm{TSS}=61.17-45.19[(\mathrm{~B} 1-\mathrm{B} 2) /(\mathrm{B} 1+\mathrm{B} 2)]$ & 0,01 & 1.40 & 37,71 & 35,55 & 26,53 & .23 \\
\hline 8 & $\mathrm{TSS}=-893.30+453.05 \cdot \mathrm{e}^{\wedge}(\mathrm{B} 1)+403.81 \cdot \mathrm{e}^{\wedge}(\mathrm{B} 2)$ & 0,67 & $27.4 * * *$ & 21,55 & 19,88 & 16,75 & .83 \\
\hline 9 & $\mathrm{TSS}=-417.65+385.76 \cdot \mathrm{e}^{\wedge}(\mathrm{B} 1+\mathrm{B} 2)$ & 0,69 & $59.22^{* * *}$ & 20,83 & 19,64 & 16,55 & .84 \\
\hline 10 & $\mathrm{TSS}=23.373+2913.27\left(\mathrm{~B} 2^{\wedge} 2\right)$ & 0,53 & $27.98^{* * *}$ & 26,36 & 24,85 & 19,69 & .73 \\
\hline 11 & $\mathrm{TSS}=-65.25+3122.35(\mathrm{~B} 1)-30654.23\left(\mathrm{~B} 2^{\wedge} 2\right)+$ & 0,54 & $10.86^{* * *}$ & 25,73 & 23,22 & 17,37 & .77 \\
\hline 12 & $\mathrm{TSS}=15.94+15553.76(\mathrm{~B} 1 \wedge 3)+10983.79(\mathrm{~B} 2 \wedge 3)$ & 0,77 & $43.86^{* * *}$ & 18,06 & 16,67 & 13,34 & .89 \\
\hline
\end{tabular}

Fuente: elaboración propia.

Nota: $r^{2}=$ coeficiente de correlación de la regresión ajustado por grados de libertad. SE = error estándar, RMSE = raíz del error cuadrático medio, MAD = desviación absoluta media, $\mathrm{cc}=$ coeficiente de correlación.

$* * * p<0.01 * p<.01$

De acuerdo con la Tabla 1, el mejor modelo fue el nro. 12:

$\mathrm{TSS}=15.94+15553.76\left(\mathrm{~b} 1^{\wedge} 3\right)+10983.79\left(\mathrm{~b} 2^{\wedge} 3\right)(9)$

En las ecuaciones (8 y 9), b1 y b2 son las reflectancias normalizadas de la banda 1 y 2 correspondientemente; este es el mejor modelo porque el RMSE fue el más bajo (16,67 mgL-1), al igual que el MAD (13,34mgL-1). Así, pues, se toman el RMSE y MAD porque evalúan la precisión de la predicción del modelo, mientras que el $r^{2}$ representa la varianza del modelo y no indica su exactitud [28]. Esto no quiere decir que sea el modelo definitivo, ya que debe cumplir con los supuestos del modelo de regresión lineal. Al verificar los supestos se encuentra que el modelo de la ecuación (6) no cumple con el supuesto de normalidad en los errores, según el test de shapiro wilk ( $\mathrm{W}=0.917, \mathrm{p}=0.039)$, pues se rechaza la hipótesis nula de normalidad en 
los errores de la regresión. Después de examinar los residuales estudentizados de la regresión se encuentra que una observación (Estación P. Martina del 28/04/2014) muestra que se trataría de un valor atípico. Por lo tanto, se excluye de la regresión, y así se soluciona el problema de normalidad en los residuos de la regresión y se llega al siguiente modelo con la misma forma funcional mostrada en la ecuación (9).

$$
\begin{gathered}
\mathrm{TSS}=12.230+16534.618\left(\mathrm{~b} 1^{\wedge} 3\right)+ \\
11124.007\left(\mathrm{~b} 2^{\wedge} 3\right) \quad(10)
\end{gathered}
$$

La ecuación (10) es el modelo definitivo en el cual b1 y b2 son las reflectancias normalizadas de las bandas 1 y 2 del sensor modis, ya sea Aqua o Terra, y Tss está dado en mgL-1.

\section{Análisis de la aplicación del modelo a las imágenes}

Para el promedio mensual, se observa para 2011 que los valores de tss en la parte norte del golfo se encuentran por debajo de los $25 \mathrm{mgL}-1$, aunque se evidencian píxeles atípicos que muestran un patrón heterogéneo y que se evidencia mucho en las imágenes diarias y promedios mensuales (Figura 8).

En la Figura 8 se identifican varios casos en los cuales los valores de tss son anormales, debido a que registran mediciones de sedimentación muy similares a las aguas de la desembocadura del Atrato en zonas de la parte norte del golfo. Esto se debe a que el modelo pierde predicción en esta zona que se considera aguas caso I (aguas donde las propiedades ópticas ya no están dominadas por los sedimentos, sino por otros factores como el fitoplancton). Sin embargo, en valores altos registrados sobre todo en la costa del municipio de Necoclí podría tener incidencia el efecto de adyacencia y la resuspensión de sedimentos desde el fondo [29]. A profundidades mayores a $50 \mathrm{~m}$ el efecto de resuspensión de los sedimentos no es un factor en la determinación de las propiedades ópticas de la sedimentación [30]. Además, la estimación cuantitativa y precisa de Tss en la región cercana a la costa es a menudo difícil, a causa de la corrección atmosférica, ya que estos algoritmos pueden fallar allí [29].

Asimismo, para mayo, septiembre y octubre se observa que la región costera de los municipios de Unguia y Acandi muestran valores de Tss altos en comparación a los otros meses, pues llegan a ser de aproximadamente 100mgL-1 en mayo, mes que muestra una estela de sedimentación alargada por el borde costero de Unguía y Acandí (ver Figura 9).

La pluma de sedimentación no es muy visible en enero, pues muestra valores muy bajos de Tss en toda la región en comparación con los demás meses. La pluma de sedimentación para 2011 tiene tres direcciones con pronunciada tendencia: la primera es en dirección norte (agosto, septiembre, octubre y noviembre); la segunda, en dirección sur, y se evidencia visualmente diciembre, y la tercera en dirección este (hacia la ciudad de turbo), para mayo y julio.
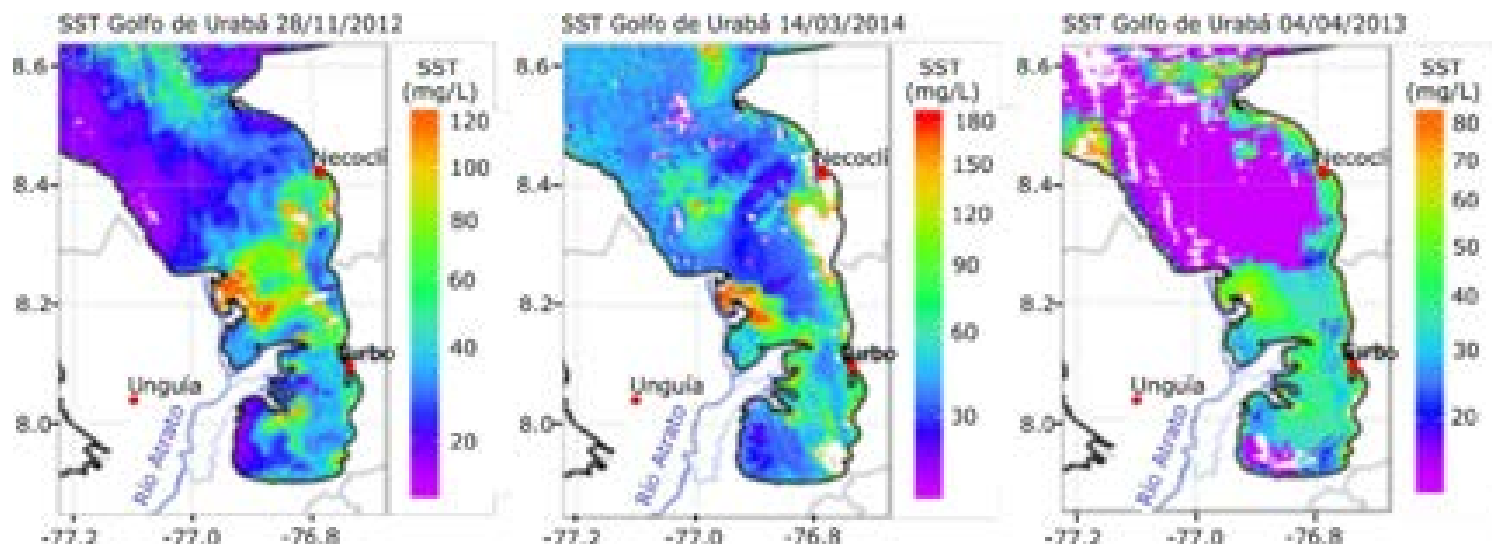

Fig. 8. Valores anormales de TSS identificados en la zona norte del golfo de Urabá Fuente: elaboración propia. 
Enero mostró valores de Tss bajos en comparación con los demás meses, pues fue inferior a los 40 mgL-1 entre 2011 y 2013. La dirección de la pluma de todas las bocas del Atrato fue en dirección sur; para 2014 y 2015 hubo un incremento de Tss en enero y llegó hasta los 70 mgL-1; mayo fue el mes que mayor heterogeneidad mostró en el promedio mensual, heterogeneidad que se evidencia en valores muy altos de Tss lejos de las bocas del Atrato, sobre todo en la parte norte del golfo, excepto para 2012. Ese año se mostró un valor máximo promedio de $100 \mathrm{mgL}-1$ y se alcanzaron valores muy altos para 2015, en comparación con mayo, lo que deja ver una pluma con alta concentración de TSS desde la boca el El Roto hasta el borde costero del municipio de Necoclí.

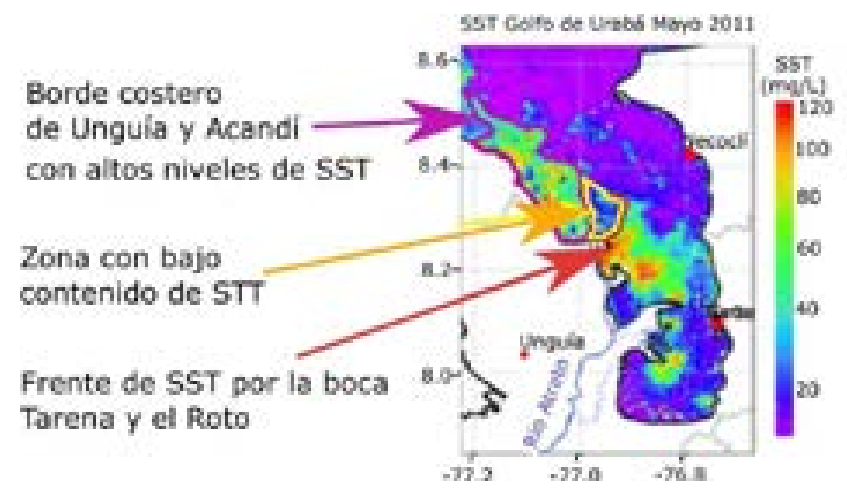

Fig. 9. Anomalía en el comportamiento de la sedimentación encontrada para el promedio mensual de mayo de 2011 Fuente: elaboración propia.

La sedimentación en bahía Colombia (ver Figura 10) está atribuida, en especial, a las descargas del río Atrato por la boca Pichindi y las del río León. Cuando bahía Colombia presenta valores bajos de Tss, por ejemplo, durante agosto y noviembre, es porque la dirección predominante de las plumas de sedimentación se encuentra hacia el norte, como se puede observar en la imagen del promedio mensual de noviembre de 2012 (Figura 10). Por el contrario, enero, febrero y marzo (en menor medida) muestran que la dirección de la pluma de sedimentación es en dirección sur, lo que ocasiona valores promedio de Tss mayores en bahía Colombia.
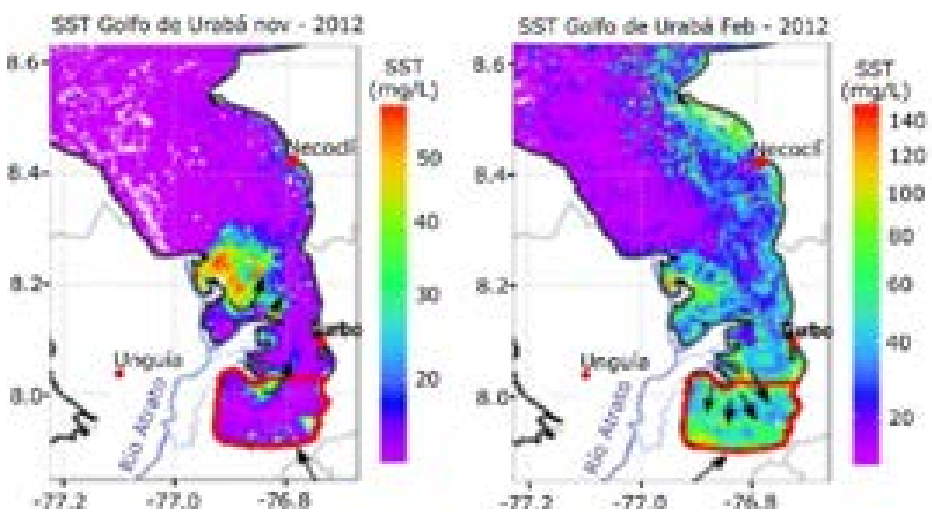

Fig. 10. Sedimentación en bahía Colombia

Fuente: elaboración propia.

Nota: en la imagen se muestra un contraste de meses con poca y mucha sedimentación en la bahía. 
La aceleración del flujo por causa de la descarga del Atrato es notoria desde bahía Colombia en el sur hasta la frontera norte, donde la pluma se recuesta preferencialmente sobre la margen oriental del golfo [11]; sin embargo, hay que tener en cuenta la influencia de la sedimentación aportada por el río León.

La dirección y forma de las plumas de sedimentación son detectables con mayor facilidad en la aplicación del modelo diario (ver Figura 11); por ejemplo, el día 22 de agosto de 2012 la pluma no tuvo dirección y el curso de los sedimentos seguía un orden radial en la salida por la boca El Roto, boca Matuntugo y boca Pichindi.
En la Figura 12 se observa que el 2 de agosto de 2011 y el 15 de noviembre de 2011 las plumas de sedimentación presentaron un comportamiento similar en dirección a Necoclí.

Otros patrones observados (ver Figura 13) permiten inducir que la fuerza con la que llega el agua en la desembocadura del Atrato al golfo de Urabá determina la dirección de la pluma de sedimentación hasta cierto lugar del golfo, cuando otras fuerzas como las mareas y el viento determinan la dirección de los sedimentos, como se observó los días 30 de agosto de 2014, 18 de noviembre de 2015 y 19 de junio de 2012. En general, en el golfo de Urabá se dan

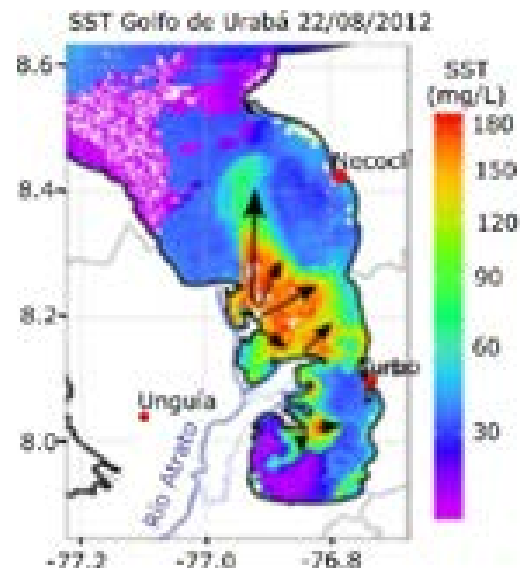

Fig. 11. Dirección de las plumas de sedimentación con forma digital en la desembocadura del río Atrato Fuente: elaboración propia.
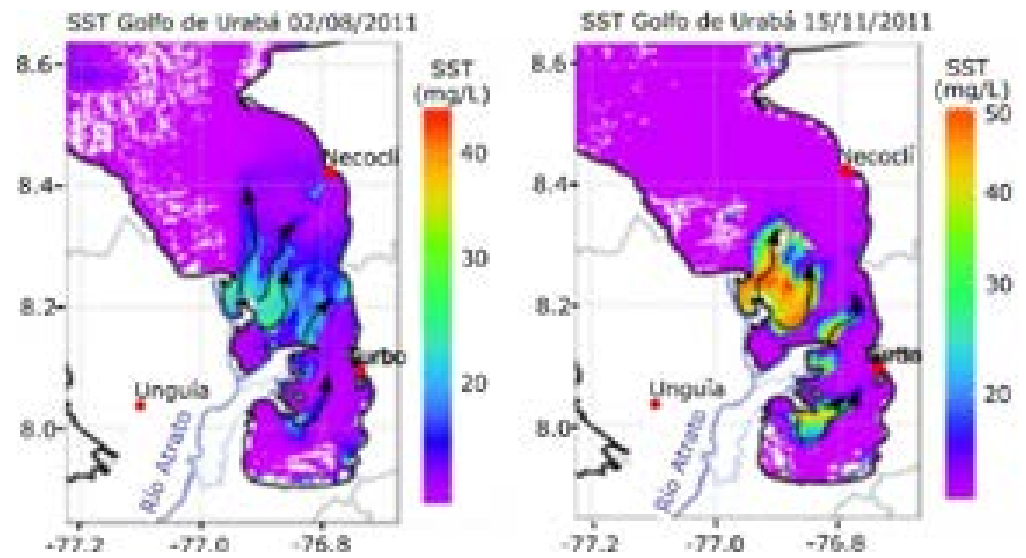

Fig. 12. Patrones de circulación de las plumas de sedimentación que muestran tendencia a seguir una trayectoria hacia el norte del golfo de Urabá

Fuente: elaboración propia. 
corrientes superficiales muy fuertes condicionadas principalmente por la entrada del río Atrato, la que a su vez se ve afectada por la dirección de los vientos y condiciona la pluma de turbidez en el golfo [31].
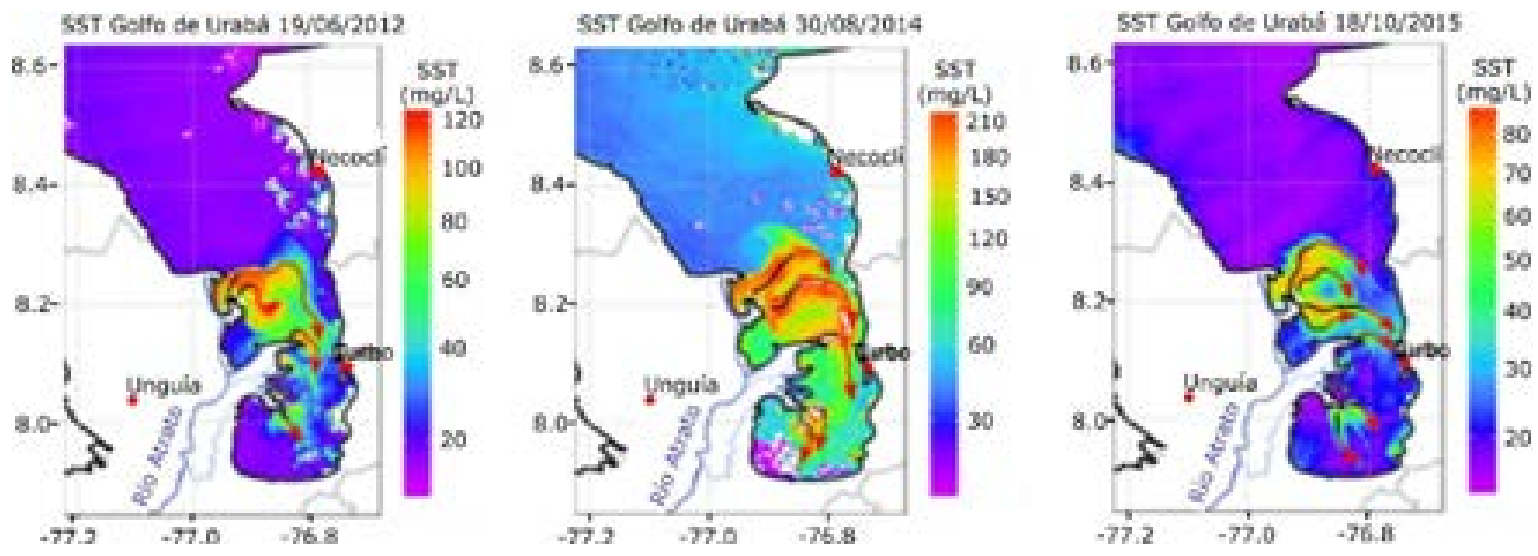

Fig. 13. Cambio de dirección en la pluma de sedimentación debida a los vientos y fuerza del agua del rio Atrato Fuente: elaboración propia.

Nota: las líneas negras identifican el curso del flujo de sedimentación gobernado por la fuerza con la que llega el agua del río al golfo; con rojo se muestra que la dirección de las plumas obedece a fuerzas como la dirección y fuerza de los vientos.

\section{Conclusiones}

Como se vio en los análisis de la aplicación del modelo a las imágenes, la concentración de sedimentos en suspensión es muy variable dentro de una amplia gama de escalas temporales y espaciales [32]. Se encontró que la zona con mayor sedimentación en el golfo de Urabá corresponde a las descargas que llegan por la boca El Roto y que se concentran en la bahía El Rotico y la bahía Yerbasal, mientras que en bahía Marirrio la sedimentación es menor en comparación con la observada en la bahía Candelaria. Este hecho se debe en gran medida a la forma que tiene la bahía que impide la acumulación de sedimentos provenientes de la boca Pichindi, los cuales, cuando se acumulan en bahía Colombia, tienden a dejarla por el borde oriental del golfo por donde los flujos sedimentarios del río León tienden a seguir. El modelo no fue óptimo en la determinación de Tss en la zona norte del golfo de Urabá, dado que en estas aguas que se consideran caso I las propiedades ópticas del agua ya no se encuentran gobernadas por los sedimentos; de allí que mucha información calculada sea de cero o presente valores muy altos de Tss comparables con los de la desembocadura del río Atrato.
La utilización de las imágenes MYD09 y MOD09 ahorra el paso de la corrección atmosférica que hoy en día es muy difícil de aplicar a las imágenes de observación del color del océano; por ende, muchos investigadores consultados proponen sus algoritmos y aplican metodologías de corrección atmosférica, que se basan en criterios subjetivos [33]. De allí que adoptar una metodología de estas pueda traer problemas para las aguas del golfo de Urabá.

El uso de un píxel a $250 \mathrm{~m}$ mejora la resolución de los procesos relativos a la variabilidad espacial, pues permiten mediciones de Tss más cerca de la orilla en comparación con la implementación de las bandas de $500 \mathrm{~m}$. Sin embargo, debido a la complejidad del medio ambiente de las aguas costeras turbias, los datos del color del océano derivados de satélites son a menudo sesgados en las regiones costeras del océano [34].

La alta resolución temporal de los datos MODIs es ventajosa en este trabajo por su capacidad de supervisar todos los días para construir los promedios mensuales y anuales [35].

La calidad de las redes de monitoreo in situ depende del número de estaciones de muestreo, 
de su representatividad espacial y la frecuencia de las mediciones [36], ya que de estas sale un insumo importante para la elaboración de los modelos de sedimentación, como también el seguimiento de las lluvias [26] que se relaciona con la cantidad de sedimentos y los vientos vinculados con la dirección de la pluma de sedimentación.

Por último, la presente investigación constituye un paso importante en la comprensión del comportamiento de la sedimentación en el golfo de Urabá a escalas diaria, semanal, mensual y anual (que hasta el día de hoy es muy poco lo que se conoce), por medio del uso de imágenes de satélite, dado que el modelo elaborado es aplicable solo a esta región del planeta. Lo anterior permite enfocar esfuerzos de las autoridades ambientales como Corporación Autónoma Regional para el Desarrollo Sostenible del Chocó (Codechocó) y Corporación para el Desarrollo Sostenible del Urabá (Corpourabá) en el cuidado y monitoreo del ecosistema marino y terrestre del golfo de Urabá. Con este estudio se identifican las zonas de mayor y menor impacto por la sedimentación, lo que permitiría realizar un seguimiento del estado de estos ecosistemas de una forma más fácil y con el fin último de su preservación y cuidado.

\section{Agradecimientos}

Al Instituto de Investigaciones Marinas y Costeras José Benito Vives de Andreis (Invemar), por los datos que el mismo instituto tomó de sólidos suspendidos totales con la Redcam, datos que fueron un insumo primordial para esta investigación.

\section{Referencias}

[1] A. Kumar, S. M. Equeenuddin, D. R. Mishra y B. C. Acharya, "Remote monitoring of sediment dynamics in a coastal lagoon: Long-term spatio-temporal variability of suspended sediment in Chilika," Estuarine, Coastal and Shelf Science, vol. 170, pp. 155-172, mar. 2016. DOI: https://doi.org/10.1016/j.ecss.2016.01.018

[2] D. Fernández-Nóvoa et al., "Analysis of the influence of river discharge and wind on the Ebro turbid plume using MODIS-Aqua and MODIS-Terra data," Journal of Marine Systems, vol. 142, pp. 40-46, feb. 2015. DOI: https://doi.org/10.1016/j.jmarsys.2014.09.009

[3] D. Doxaran et al., "Retrieval of the seawater reflectance for suspended solids monitoring in the East China Sea using MODIS, MERIS and GOCI satellite data," Remote Sensing Environment, vol. 146, pp. 36-48, abr. 2014. DOI: https://doi.org/10.1016/j.rse.2013.06.020

[4] C. M. Long y T. M. Pavelsky, "Remote sensing of suspended sediment concentration and hydrologic connectivity in a complex wetland environment," Remote Sensing Environment, vol. 129, pp. 197-209, feb. 2013. DOI: https://doi.org/10.1016/j.rse.2012.10.019

[5] J. Chen, W. Quan, T. Cui y Q. Song, "Estimation of total suspended matter concentration from MODIS data using a neural network model in the China eastern coastal zone," Estuarine, Coastal and Shelf Science, vol. 155, pp. 104-113, mar. 2015. DOI: https://doi.org/10.1016/j. ecss.2015.01.018

[6] G. Wu et al., "Comparison of MODIS-based models for retrieving suspended particulate matter concentrations in Poyang Lake, China," International Journal of Applied Earth Observation and Geoinformation, vol. 24, pp. 63-72, oct. 2013. DOI: https://doi.org/10.1016/j.jag.2013.03.001

[7] J.-E. Min, J.-H. Ryu, S. Lee y S. Son, "Monitoring of suspended sediment variation using Landsat and MODIS in the Saemangeum coastal area of Korea," Marine Pollution Bulletin, vol. 64, no. 2, pp. 382-390, feb. 2012. DOI: https://doi.org/10.1016/j.marpolbul.2011.10.025

[8] M. Zhang, J. Tang, Q. Dong, Q. Song y J. Ding, "Retrieval of total suspended matter concentration in the Yellow and East China Seas from MODIS imagery," Remote Sensing Environment, vol. 114, no. 2, pp. 392-403, feb. 2010. DOI: https://doi.org/10.1016/j.rse.2009.09.016

[9] Y. Sagarminaga, G. Chust y D. Dailloux, "Detección y seguimiento de la pluma del río Adour mediante Modis," en XI Congreso Nacional de Teledetección, Puerto de la Cruz. Tenerife, España, 2005, pp. 227-230.

[10] A. G. Dekker, R. J. Vos y S. W. M. Peters, “Comparison of remote sensing data, model results and in situ data for total suspended matter (TSM) in the southern Frisian lakes," Science of the Total Environment, vol. 268, no. 1-3, pp. 197-214, mar. 2001. DOI: https://doi.org/10.1016/ S0048-9697(00)00679-3

[11] S. Escobar y A. Carlos, "Relevancia de procesos costeros en la hidrodinámica del golfo de Urabá (caribe colombiano)," Bolletin of Marine and Coastal Research, vol. 40, no. 2, pp. 327-346, dic. 2011. DOI: https://doi. org/10.25268/bimc.invemar.2011.40.2.114

[12]R. A. Gómez. Los mares mexicanos a través de la percepción remota. Ciudad de México, México: Plaza y Valdés, 2001.

[13] C. García y P. C. Sierra. Atlas del golfo de Urabá: una mirada al Caribe de Antioquia y Chocó. Santa Marta, 
Colombia: Instituto de investigaciones Marinas y Costeras (Invemar) y Gobernación de Antioquia, 2007.

[14] E. F. Vermote, J. C. Roger y J. P. Ray, "MODIS Surface Reflectance User's Guide”, 2015. [En línea]. Disponible en: http://modis-sr.ltdri.org/guide/MOD09_UserGuide_v1.4.pdf

[15]P. E. Tarrant, J. A. Amacher y S. Neuer, "Assessing the potential of Medium-Resolution Imaging Spectrometer (MERIS) and Moderate-Resolution Imaging Spectroradiometer (MODIS) data for monitoring total suspended matter in small and intermediate sized lakes and reservoirs," Water Resourses Research, vol. 46, no. 9, p. W09532, sep. 2010. DOI: https://doi. org/10.1029/2009WR008709

[16] M. S. Wong et al., "Modeling of Suspended Solids and Sea Surface Salinity in Hong Kong using Aqua/MODIS Satellite Images," Korean Journal Remote Sensing, vol. 23, no. 3, p. 8, 2007.

[17] J. A. Concha y J. R. Schott, "In-water component retrieval over Case 2 water using Landsat 8: Initial results," en 2014 IEEE Geoscience and Remote Sensing Symposium, 2014, pp. 4458-4461. DOI: https://doi.org/10.1109/IGARSS.2014.6947481

[18] Instituto de Investigaciones Marinas y Costeras José Benito Vives de Andreis (Invemar), "Informe del estado de los ambientes y recursos marinos y costeros en colombia año 2014", no. 3, 2015. [En línea]. Disponible en: http://www.invemar.org.co/redcostera1/invemar/ docs/ier2014.pdf

[19] Instituto de Investigaciones Marinas y Costeras José Benito Vives de Andreis (Invemar) y Red de Vigilancia para la Conservación y Protección de las Aguas Marinas y Costeras de Colombia (Redcam), "Datos históricos del monitoreo de la Red de Vigilancia para la conservación y protección de la calidad de las aguas marinas y costeras de Colombia - Redcam en el Golfo de Urabá," Sistema de Información Ambiental Marina de Colombia (SIAM) y Redcam, mar. 2016.

[20] G. Wu, L. Liu, F. Chen y T. Fei, "Developing MODISbased retrieval models of suspended particulate matter concentration in Dongting Lake, China," International Journal of Applied Earth Observation and Geoinformation, vol. 32, pp. 46-53, oct. 2014. DOI: ttps://doi. org/10.1016/j.jag.2014.03.025

[21] L. Feng, C. Hu, X. Chen, L. Tian y L. Chen, "Human induced turbidity changes in Poyang Lake between 2000 and 2010: Observations from MODIS," Journal of Geophysical Research, vol. 117, no. C7, p. C07006, jul. 2012. DOI: https://doi.org/10.1029/2011JC007864

[22] D. Doxaran, J.-M. Froidefond, P. Castaing, y M. Babin, "Dynamics of the turbidity maximum zone in a macro- tidal estuary (the Gironde, France): Observations from field and MODIS satellite data," Estuarine, Coastal and Shelf Science, vol. 81, no. 3, pp. 321-332, feb. 2009. DOI: https://doi.org/10.1016/j.ecss.2008.11.013

[23] T. M. Pavelsky y L. C. Smith, "Remote sensing of suspended sediment concentration, flow velocity, and lake recharge in the Peace-Athabasca Delta, Canada," Water Resourses Research, vol. 45, no. 11, p. W11417, nov. 2009. DOI: https://doi.org/10.1029/2008WR007424

[24] Z. Chen, C. Hu y F. Muller-Karger, "Monitoring turbidity in Tampa Bay using MODIS/Aqua 250-m imagery," Remote Sensing of Environment, vol. 109, no. 2, pp. 207-220, jul. 2007. DOI: https://doi.org/10.1016/j.rse.2006.12.019

[25]E. Park y E. M. Latrubesse, "Modeling suspended sediment distribution patterns of the Amazon River using MODIS data," Remote Sensing of Environment, vol. 147, pp. 232-242, may. 2014. DOI: https://doi.org/10.1016/j. rse.2014.03.013

[26] Y. Zhang, K. Shi, Y. Zhou, X. Liu y B. Qin, "Monitoring the river plume induced by heavy rainfall events in large, shallow, Lake Taihu using MODIS $250 \mathrm{~m}$ imagery," Remote Sensing Environment, vol. 173, pp. 109-121, feb. 2016. https://doi.org/10.1016/j.rse.2015.11.020

[27] K. Shi et al., "Long-term remote monitoring of total suspended matter concentration in Lake Taihu using 250 m MODIS-Aqua data," Remote Sensing Environment, vol. 164, pp. 43-56, jul. 2015. https://doi.org/10.1016/j. rse.2015.02.029

[28] N. Bernardo, F. Watanabe, T. Rodrigues y E. Alcântara, "Evaluation of the suitability of MODIS, OLCI and OLI for mapping the distribution of total suspended matter in the Barra Bonita Reservoir (Tietê River, Brazil)," Remote Sensing Applications: Societu and Environment, vol. 4, pp. 68-82, oct. 2016. DOI: https://doi. org/10.1016/j.rsase.2016.06.002

[29] I. Caballero, J. Ruiz y G. Navarro, "Dynamics of the turbidity plume in the Guadalquivir estuary (SW Spain): A remote sensing approach," en OCEANS 2011 IEEE, Santander, España, 2011, pp. 1-11. DOI: https:// doi.org/10.1109/Oceans-Spain.2011.6003489

[30] M. Zhang, Q. Dong, T. Cui y J. Ding, "Remote Sensing of Spatiotemporal Variation of Apparent Optical Properties in Bohai Sea," IEEE Journal of Selected Topics in Applied Earth Observations and Remote Sensing, vol. 8, no. 3, pp. 1176-1184, mar. 2015. DOI: https://doi.org/10.1109/JSTARS.2014.2380785

[31] A. L. Álvarez y G. F. Bernal, “Estimación del campo de transporte neto de sedimentos en el fondo de bahía Colombia con base en análisis de tendencia del tamaño de grano", Avances en recursos Hidráulicos, no. 16, pp. 41-50, 2009. 
[32] R. L. Miller, R. L. y B. A., "McKee Using MODIS Terra $250 \mathrm{~m}$ imagery to map concentrations of total suspended matter in coastal waters," Remote Sensing of Environment, vol. 93, no. 1-2, pp. 259-266, 2004. DOI: https://doi.org/10.1016/j.rse.2004.07.012

[33]E. Kaba, W. Philpot y T. Steenhuis, "Evaluating suitability of MODIS-Terra images for reproducing historic sediment concentrations in water bodies: Lake Tana, Ethiopia," International Journal of Applied Earth Observation and Geoinformation, vol. 26, pp. 286-297, feb. 2014. https://doi.org/10.1016/j.jag.2013.08.001

[34] W. Shi y M. Wang, "Satellite observations of flood-driven Mississippi River plume in the spring of 2008," Geophysical Research Letters, vol. 36, no. 7, p. L07607, abr. 2009. DOI: https://doi.org/10.1029/2009GL037210
[35] S. Wang, Y. Jiao, Y. Zhou y L. Wang, "Determination of suspended sediment concentration of Taihu Lake based on season difference using multi-temporal MODIS image data," en 2007 IEEE International Geoscience and Remote Sensing Symposium, 2007, pp. 4570-4573. DOI: https://doi.org/10.1109/IGARSS.2007.4423874

[36] E. Robert, M. Grippa, L. Kergoat, S. Pinet, L. Gal, G. Cochonneau y J.-M. Martínez. "Monitoring water turbidity and surface suspended sediment concentration of the Bagre Reservoir (Burkina Faso) using MODIS and field reflectance data," International Journal of Applied Earth Observation and Geoinformation, vol. 52, 243-251, 2016. DOI: https://doi. org/10.1016/j.jag.2016.06.016 